\title{
Libéralisation et secteur informel
}

Réflexions à partir du cas mexicain

Economic liberalization and the informal sector : Reflections from the Mexican case

\section{Carlos Alba et Pascal Labazée}

\section{OpenEdition \\ Journals}

Édition électronique

URL : http://journals.openedition.org/transcontinentales/610

DOI : 10.4000/transcontinentales. 610

ISBN : 978-2-7351-1565-5

ISSN : 1775-397X

Éditeur

Editions de la maison des sciences de l'homme

\section{Édition imprimée}

Date de publication : 1 juin 2007

Pagination : 77-98

ISBN : 978-2-200-92396-9

ISSN : 1950-1684

\section{Référence électronique}

Carlos Alba et Pascal Labazée, «Libéralisation et secteur informel », Transcontinentales [En ligne], 4 2007, document 5, mis en ligne le 28 avril 2011, consulté le 08 septembre 2020. URL : http:// journals.openedition.org/transcontinentales/610 ; DOI : https://doi.org/10.4000/transcontinentales. 610 


\title{
Libéralisation et secteur informel
}

\author{
Réflexions à partir du cas mexicain
}

\author{
Carlos Alba et Pascal Labazée
}

La part croissante prise par les activités et l'emploi dans le secteur informel au cours des décennies d'ajustement, d'ouverture et de libéralisation dans les économies en développement est un fait relativement bien connu et documenté, la généralité et l'ampleur de ce phénomène restant partiellement mesurées en raison, notamment, de la disparité des définitions de l'informalité, et des difficultés concrètes à inventorier les unités qui en font partie. En s'appuyant, il y a peu, sur une recension intercontinentale des données disponibles dans ce domaine, l'Organisation internationale du travail concluait que "la majeure partie des emplois nouveaux des dernières années, particulièrement dans les pays en développement et en transition, avaient été créés dans l'économie informelle ${ }^{1}$ ». Tel est bien le cas dans les pays d'Amérique latine où la décennie 1990 a été marquée par un double changement dans la structure d'emploi tenant, d'une part, à la vive informalisation des postes de travail nouveaux - en ville, deux créations sur trois ont été le fait d'établissements de moins de cinq travailleurs - et, d'autre part, à la tertiarisation des activités, les nouveaux arrivants s'insérant plutôt dans le commerce et les services ${ }^{2}$. Sauf exceptions - Argentine, Chili notamment - , la tertiarisation des emplois vient principalement de la multiplication des micro-unités, et plus marginalement de l'expansion des entreprises moyennes et grandes du secteur moderne. Cette dynamique semble en outre peu sensible aux variations conjoncturelles des économies nationales: sauf dans de rares cas où la hausse annuelle du produit intérieur s'est révélée forte

1 - El trabajo decente y la economía informal, Ginebra, Oficina Internacional del Trabajo (OIT), Informe IV, 2002.

2 - Une décennie de développement social en Amérique latine, Santiago du Chili, Nations uniesCEPAL-IHEAL, Livre de la CEPALC n 77, 2004, p. 136-138.

Carlos Alba : El Colegio de México.

Pascal Labazée : Institut de recherche pour le développement (IRD) - El Colegio de México. 
et répétée, l'informalisation ne reculerait pas en dessous d'un taux de croissance substantiel, de l'ordre de 3,5\% l'an ${ }^{3}$.

Dans ce texte, la dynamique contemporaine du secteur informel au Mexique sera traitée sous l'angle des rapports qu'il entretient avec les mutations politiques et économiques des deux dernières décennies, l'hypothèse étant que l'expansion de certains types d'activités, liée à l'expansion de nouveaux circuits marchands internationaux ayant recours à la fraude, est un facteur central, quoique peu étudié, dans l'apparition des nouvelles configurations institutionnelles qui accompagnent les politiques de libéralisation. On s'intéressera particulièrement au petit commerce de rue, dont la prolifération a été notée dans la plupart des grandes villes latino-américaines et qui se situe désormais à la charnière de trois problématiques distinctes - contribution à la croissance de l'informel, participation à la diffusion de produits de contrebande et de contrefaçon, rôle politique des organisations de vendeurs dans des systèmes de compétition ouverte entre partis -, dont les déterminations réciproques sont de nature à transformer les approches de l'informalité, notamment la fonction que celle-ci occupe dans les nouveaux modes de croissance, au Mexique comme ailleurs. Les fonctions sociales, économiques et politiques dévolues à l'informalité, non à la périphérie mais au coeur de dynamiques productives orientées vers l'extérieur, rendent en effet définitivement inopérantes les approches en termes de marginalité, de dépendance, de dualité, etc. ${ }^{4}$, et passablement ambiguës les interprétations qui y voient le produit de l'inventivité des travailleurs pauvres ou de stratégies dites «desde abajo [depuis la base] ${ }^{5}$ ». Elles questionnent aussi le degré de validité des analyses contracycliques faisant de l'informel un secteur essentiellement réactif, absorbant une partie des variations de conjoncture et des chocs du secteur moderne ${ }^{6}$, dans la mesure où, simultanément, les micro-unités de commerce impliquent les industries nationales dans des rapports de forces fortement dissymétriques entre pays, ou biaisés par diverses pratiques - dumping, contrebande, subventions à l'exportation, etc. Elles piègent aussi les débats concernant la légalisation des micro-entreprises de ce secteur, qu'elle soit entendue, à la manière de Ghersi et de Soto ${ }^{7}$, comme un objectif pour réduire les coûts d'accès au secteur moderne, ou comme le moyen d'éradiquer une

3 - J.-A. Ocampo et J. Martin (dir.), Une décennie d'ombres et de lumières. L'Amérique latine et les Caraibes dans les années 1990, Santiago du Chili, CEPALC-IHEAL, 2003, p. 249.

4 - On trouvera une révision de la littérature consacrée à ces approches dans l'article de C. Alba et D. Kruijt, "El significado del sector informal y la microempresa en América latina y en México", in T. Calvo et B. Méndez (dir.), Micro y pequeña empresa en México. Frente a los retos de la globalización, México, Misceláneas, 1995.

5 - Ces approches perdent de leur pouvoir explicatif dès lors qu'une part croissante du secteur informel se trouve insérée, notamment comme agents de la distribution finale, dans des circuits de production et de distribution aux ramifications internationales.

6 - Amplement documentées, par exemple par Kelley («The Informal Sector and the Macroeconomy: A CGE Approach for Peru», World Development, vol.22, n 9,.1994) pour le Pérou, ou Montaud (Deux approches macroéconomiques du secteur informel en Équateur, Bordeaux, CED-DT n ${ }^{\circ} 47$, 2000) pour l'Équateur, ces analyses convergent vers le constat d'un rôle tendanciellement contracyclique de l'ensemble du secteur; ce qui n'exclut nullement que certains segments de ce même secteur s'appuient sur des dynamiques indépendantes des évolutions du secteur moderne.

7 - H. de Soto, E. Ghersi, M. Ghibellini et Instituto Libertad y Democracia, El otro sendero : la revolución informal, México, Editorial Diana, 1987. 
fracture jugée artificielle et dont les causes seraient moins objectives que conceptuelles $^{8}$. Enfin, le nombre et la concentration spatiale des ambulants dans les villes, signe des difficultés de l'économie ouverte à créer des emplois salariés, leur confère un rôle important dans les luttes politiques locales voire nationales, le plus souvent en tant qu'acteurs dominés dans des systèmes de type clientéliste ou corporatiste.

Dans une première partie, on exposera quelques traits caractéristiques de la progression du secteur informel au Mexique, en suggérant l'importance de certains " complexes d'activités » - articulation de fonctions productives et commerciales visant à répondre à un petit nombre de demandes de base des populations citadines. Dans une seconde partie, on abordera les effets différenciés des politiques d'ouverture et de libéralisation sur les micro-unités de production et sur les petits commerces, afin d'expliquer les raisons du déclin numérique des premières et, symétriquement, de la hausse du poids relatif des seconds. On évoquera en troisième partie l'essor des biens de consommation courante issus de la contrebande et de la contrefaçon au cours des années 1980 et 1990, via l'apparition de réseaux d'opérateurs extrêmement diversifiés et dont certains intègrent, dans leurs circuits de distribution finale, une fraction non négligeable de micro-opérateurs urbains. Le texte esquisse ainsi l'hypothèse d'une progressive dilution des frontières entre l'informalité et l'illégalité dans un contexte de mondialisation, et du renforcement du rôle des réseaux transnationaux dans le contrôle de l'illégalité et l'approvisionnement local.

\section{L'informel dans l'économie mexicaine: de l'ouverture à l'expansion des micro-activités marchandes}

Comme tous les autres pays d'Amérique latine, le Mexique, qui a opté dans les années 1980 pour un mode d'industrialisation orienté vers l'extérieur et fondé en grande partie sur l'expansion territoriale de la maquiladora [industrie de sous-traitance] d'exportation, a connu une nette progression des micro-activités urbaines au cours des deux dernières décennies. En 2004, celles-ci absorbaient en effet 44,9\% du total de la population occupée dans les agglomérations de plus de 100000 habitants, contre seulement 40,3\% en $1990^{9}$, ce qui représenterait environ 2,4 millions d'emplois supplémentaires générés par les micro-unités des villes moyennes et grandes du pays. Ce constat tend à confirmer que l'industrie maquiladora ne s'est pas imposée comme le principal moteur de la création d'emplois au plan national, même pendant les années où ses activités ont été en forte croissance ${ }^{10}$, et qu'elle ne suffit pas à accueillir les nouveaux entrants sur le marché du travail. Si les cinq années ayant suivi la signature du Traité de libre commerce d'Amérique du Nord ont été marquées par un doublement du nombre des travailleurs dans la maquiladora d'exportation, la situation s'est détériorée à partir des années 2000 sous l'effet de divers facteurs - conjoncture défavorable de l'économie

8 - J. Monnet, «El prisma del ambulantaje. Conceptualización del ambulantaje, de los vendedores a los clientes : un acercamiento a la métrópoli postfordista», Séminaire El Ambulantaje en la Ciudad de México, México, CEMCA-Universidad Nacional Autónoma de México, 2006

9 - C. Salas et E. Zepeda, "Ocupación e ingresos en México : 2000-2004», in E. de la Garza et C. Salas (coord.), La situación del trabajo en México, México, Plaza y Valdés, 2006.

10 - M. de la Martínez, «La industria maquiladora en México», in E. de la Garza et C. Salas, op. cit., 2006. 
nord-américaine, cours élevé du peso, délocalisations au profit des pays d'Amérique centrale, progression spectaculaire des importations asiatiques par les États-Unis, etc. ${ }^{11}$. En s'en tenant aux agglomérations industrielles du District fédéral et de l'État de Mexico, qui représentent encore $29,6 \%$ de la production manufacturière nationale ${ }^{12}, 54 \%$ des emplois créés entre 1996 et 2002 sont localisés dans des établissements de cinq personnes au plus, la part revenant aux unités comptant un seul travailleur en représentant plus de la moitié ${ }^{13}$. Dans les seuls secteurs du commerce et des services, les micro-entreprises situées dans ces agglomérations ont généré plus de 370000 emplois nouveaux pendant cette période, devançant largement la croissance des micro-unités de production (49000 emplois) et de tout autre type de petites activités. Il en va de même dans les autres grandes villes industrielles du pays, où la part des actifs occupés dans les unités de moins de cinq travailleurs a régulièrement progressé dans les dix dernières années, l'accélération étant nette depuis le début de cette décennie. Qu'il s'agisse des plus anciennes métropoles du pays ayant porté l'industrialisation à l'ère de la substitution aux importations - Puebla, Guadalajara ou León par exemple -, des villes du Nord accueillant plutôt les industries maquiladoras, ou de celles du Sud-Est où le poids du secteur privé manufacturier a toujours été modeste, la quasi-totalité des agglomérations urbaines du Mexique a connu une accélération spectaculaire du poids des micro-unités dans l'emploi local, notamment depuis l'an $2000^{14}$, liée pour l'essentiel à la croissance du nombre de petits commerces et d'unités de services à caractère familial.

L'essor du monde des micro-unités urbaines mexicaines ne résulte pas, loin s'en faut, d'une croissance homogène des divers segments qui le composent. La dynamique d'ensemble est en effet tirée par un petit nombre d'activités intégrées dans quatre "complexes» visant à satisfaire les principales demandes de base des ménages citadins : se nourrir, se vêtir, se loger, se déplacer ${ }^{15}$. Une recomposition

11 - C. Alba, «L'industrie maquiladora dans le contexte des transformations régionales", in Y.-A. Fauré, L. Kennedy et P. Labazée, Productions locales et marché mondial dans les pays émergents. Brésil, Inde, Mexique, Paris, IRD-Karthala, 2005.

12 - Données INEGI (Instituto Nacional de Estadística, Geografía e Informática), 2006 : Sistema de Cuentas Nacionales de México, PIB por Entidad Federativa 1999-2004, Aguascalientes, INEGI.

13 - À partir des données INEGI, Encuesta nacional de empleo, Distrito Federal et Estado de México, Ediciones 1996, 2002, Aguascalientes, INEGI-Secretaría del Trabajo y Previsión social.

14 - La part de l'emploi urbain dans ces entreprises est ainsi passée de 41,6 \% à 45,2 \% à Guadalajara entre 2000 et 2004, de $41 \%$ à 49,2\% à Puebla, de 36,9\% à 41,1\% à León (Guanajuato), de 30,8 \% à $33,1 \%$ à Chihuahua, de $34 \%$ à 40,9 \% à Hermosillo, de 33,4\% à 40,3\% à Tijuana, etc. Les villes de la région sud-est ont aussi connu une même croissance de l'informel - de 42,5\% à 49,1\% à Veracruz par exemple. Les seules exceptions, liées à des dynamiques d'emploi spécifiques à l'échelon local, sont les villes de Monterrey - de 34,4 \% à 34,7 \% aux dates indiquées - et Querétaro - 41,7 \% à $40 \%$. Données calculées à partir de INEGI 2006, Encuesta nacional de empleo urbano (ENEU), Series históricas, Aguascalientes, INEGI.

15 - On reprend ici une notion élaborée par J.-M. Cour (Les enjeux de l'urbanisation dans les pays en voie de peuplement, Paris, OCDE-Club du Sahel, 1995) qui, bien qu'ayant été forgée dans et pour un autre contexte continental, rend bien compte, d'une part, de la concentration des activités autour d'un petit nombre de demandes urbaines, d'autre part, de l'organisation et de la répartition spécifique de ces micro-activités au sein de chacun des complexes. La seule différence notable entre les contextes africains et latino-américains consiste dans le poids relatif de ces "complexes» au sein des activités informelles - il est plus élevé dans les grandes et moyennes villes d'Afrique, puisque concentrant plus de $85 \%$ des micro-entreprises (Y.-A. Fauré et P. Labazée, Socio-économie des villes africaines, Paris, Karthala, 2002) - et dans la pondération entre ces complexes - le poids des activités orientées vers l'alimentation apparaît moins élevé en Amérique latine, au profit de celles se consacrant au transport, et à la construction, à l'entretien et à l'équipement du logement. 
des données de la Encuesta Nacional de Micronegocios ${ }^{16}$ indique ainsi qu'en 2002, près de deux micro-unités sur trois faisaient partie de l'un de ces quatre complexes, proportion en légère hausse par rapport à 1994 (voir tableau 1) ${ }^{17}$. Situé au coeur du secteur informel ${ }^{18}$, le complexe "alimentation" concentre plus du quart des petits établissements du pays, qu'ils se destinent à la mise en marché de biens primaires non transformés, à la vente de produits de l'industrie agro-alimentaire, ou aux activités de préparation alimentaire et de petite restauration populaire, ces dernières ayant enregistré une croissance particulièrement élevée dans les dix dernières années. Les établissements de production et de vente de textiles, vêtements, chaussures et accessoires divers de mode représentent pour leur part près de $10 \%$ des micro-entreprises du pays, le segment commercial de ce complexe ayant enregistré la croissance la plus élevée de toutes les micro-activités sur la période 1994-2002, tandis que le nombre d'ateliers de production de vêtements et de chaussures tendait, à l'inverse, à régresser. Les unités du complexe "logement et équipement des ménages", soit $20 \%$ des micro-établissements du pays, ont progressé en nombre, en particulier les divers corps de métiers du bâtiment - maçons, électriciens, plombiers, charpentiers, etc.; 80 \% de ces établissements, dont le marché principal est articulé à l'autoconstruction dans les quartiers populaires, n'occupent qu'un seul travailleur. Enfin, le «complexe» des activités orientées vers le transport de marchandises et de voyageurs, ainsi que vers la réparation des véhicules, représente près de $11 \%$ des entreprises du secteur informel.

TABLEAU $1:$ ÉVOLUTION DU NOMBRE DES MICRO-UNITÉS
DES PRINCIPAUX «COMPLEXES D'ACTIVITÉS» (1992-2002)

\begin{tabular}{l|c|c|c|c|c|}
\hline & \multicolumn{2}{|c|}{1994} & \multicolumn{2}{c|}{2002} & $1994-2002$ \\
\hline Total micro-unités & 3090243 & 100,0 & 4414600 & 100,0 & $+42,9$ \\
\hline Dont : & & & & & \\
$\quad$ Alimentation & 851395 & 27,6 & 1156171 & 26,2 & 35,8 \\
$\quad$ Logement et équipement & 541990 & 17,5 & 849584 & 19,2 & 56,7 \\
Textiles, vêtements et divers & 277404 & 9,0 & 418659 & 9,5 & 50,9 \\
$\quad$ Transport & 322609 & 10,4 & 481624 & 10,9 & 49,3 \\
\hline Ensemble & 1993398 & 64,5 & 2906038 & 65,8 & 45,7
\end{tabular}

Sources : À partir de ENAMIN, 1994, 2002 (INEGI).

Deux aspects majeurs des mutations touchant la structure des micro-activités urbaines méritent par ailleurs d'être évoqués, qui étayent l'hypothèse d'une rupture

16 - Encuesta Nacional de Micronegocios, Aguascalientes, Instituto Nacional de Estadística, Geografía e Informática (INEGI), 1994, 2002.

17 - Les données de l'ENAMIN sont représentatives des aires urbaines de plus de 100000 habitants, et permettent de disposer d'informations générales sur les micro-établissements sans local et sans enregistrement administratif.

18 - J. L. Álvarez Galván et C. Tilly, «Trabajadores en el comercio y los servicios en México : trabajo marginal», in E. de la Garza et C. Salas (coord.), op. cit., 2006. 
dans les dynamiques antérieurement associées à l'informalité et, simultanément, d'une consolidation de formes nouvelles d'articulation au marché national et mondial. Le premier réside dans le tassement du nombre et du poids relatif des petits ateliers de production en général, et plus particulièrement de ceux appartenant aux complexes "alimentation" et "textiles, vêtements et divers». La chute du nombre des petits établissements a été, respectivement pour ces complexes, de 25000 et 16000 entre 1994 et 2002, alors même que les micro-unités commercialisant ces mêmes biens enregistraient les plus fortes croissances de l'ensemble du secteur informel à la même période. Cet apparent paradoxe tient au fait que l'approvisionnement de ces complexes s'est profondément modifié à la suite des politiques d'ouverture commerciale, dans le premier cas par le poids croissant des grands groupes de l'agro-alimentaire dans divers produits clés de la consommation urbaine, y compris populaire et de rue, ainsi que par la hausse des importations sur divers biens de base auparavant élaborés par des petits établissements nationaux ${ }^{19}$; dans le second cas par la progression des importations de vêtements et chaussures à bas prix venant le plus souvent de divers pays asiatiques ${ }^{20}$, dont l'effet dissolutif sur les petits ateliers de divers pôles du pays a été étudié ailleurs ${ }^{21}$. En d'autres termes, les micro-unités de production résistent difficilement aux compétitions nationales et étrangères sur les biens à faible prix unitaire, tandis que la prolifération des petits commerces favorise la distribution à moindre coût des importations, légales et illégales : c'est précisément dans la vente au détail des biens alimentaires et des vêtements, où la compétition entre micro-établissements est la plus vive, que les niveaux moyens de rémunération ont le moins progressé dans les quinze dernières années ${ }^{22}$. Le second aspect réside dans l'essor des micro-activités rémunératrices réalisées sans local - commerce de rue, marchés et tianguis ${ }^{23}$, colportage notamment -, qui se sont imposées comme la modalité dominante de l'informel urbain contemporain puisqu'elles représentent $55 \%$ et $64 \%$ respectivement des établissements marchands et de services. L'ambulantage, terme désignant habituellement cet ensemble varié d'activités exercées sans local, semble désormais partiellement pertinent, la présence des points de vente sur la voie publique étant devenue permanente ${ }^{24}$ et tolérée, pour des raisons d'ordre politique, par les pouvoirs locaux des grandes villes.

Sont associées à l'essor des activités sans local bon nombre de caractéristiques qui en soulignent la précarité. Parmi elles, la progression considérable de la part des emplois familiaux qui concernent 53,2 \% de l'ensemble des micro-établissements

19 - Sur l'intérêt porté par l'industrie agro-alimentaire envers la consommation populaire, notamment de rue, on se reportera aux diverses études du Fondo de Capitalización e Inversión del Sector Rural (FOCIR), notamment : Transformación de la agroindustría y del comercio internacional de los alimentos en México, México, AIC-FOCIR, 2005.

20 - E. Dussel et L. Dong, Oportunidades y retos económicos de China para México y Centroamérica, México, Naciones Unidas-CEPAL, 2004.

21 - M. Estrada et P. Labazée, "Transformaciones productivas en el Estado de Guanajuato. Empresarios locales y globalización", in M. Estrada et P. Labazée (dir.), Globalidad y localidad. Producción, actores, migraciones e identidades, CIESAS-IRD-Casa Chata, 2007.

22 - J. L. Álvarez Galván et C. Tilly, art. cit., 2006; Censo Económico, Aguascalientes, Instituto Nacional de Estadística, Geografía e Informática (INEGI), 1984, 2004.

23 - Les tianguis sont des marchés populaires mexicains.

24 - J. Monnet, art. cit., 2006. 
du pays en 2002 contre 41,7 \% en 1994, les petits commerces étant les plus utilisateurs de main-d'œuvre domestique à très faible niveau de rémunération ${ }^{25}$; l'absence d'enregistrement administratif pour $77,4 \%$ des points de vente sans local - 61,6 \% en 1994 -, cette dernière caractéristique étant associée à une montée en puissance des organisations de vendeurs de rue qui tendent à s'imposer en intermédiaires entre les petits opérateurs et les services administratifs et fiscaux, par exemple dans la vente de vêtements ${ }^{26}$ et dans la petite distribution alimentaire. Enfin, comme dans d'autres pays latino-américains, il a été démontré que le commerce ambulant tendait à se concentrer dans les centres des principales villes du pays ${ }^{27}$, suscitant partout des tensions sur l'organisation d'un espace par nature restreint et une précarité croissante des petits opérateurs envers les politiques publiques d'aménagement des centres urbains et de régulation de l'ambulantage ${ }^{28}$. Dans le cas de la ville de Mexico, la tendance au regroupement des vendeurs de rue dans le centre historique renvoie à la place particulière occupée par ce quartier dans le processus de concentration des activités économiques dans la capitale du pays. Ainsi, des années 1930 à 1970, l'expansion industrielle a été localisée dans diverses zones du Nord de la ville, la croissance commerciale ayant bénéficié au centre historique, site depuis toujours occupé par des magasins de gros et des entrepôts dont le nombre progressa fortement sous le Porfiriat ${ }^{29}$. Cette dynamique a de plus été accompagnée par des dotations d'infrastructures favorisant les déplacements humains de la périphérie vers le centre de la mégapole, notamment via l'aménagement de dessertes routières et de métro. Toutefois, à compter des années 1950, la saturation du quartier en implantations marchandes, culturelles et administratives a suscité un processus, nullement maîtrisé par les autorités locales, de déconcentration résidentielle, de délocalisation des institutions culturelles et politiques, d'essaimage d'une partie des grands magasins vers les quartiers semi-résidentiels de la ville, enfin de départ des sièges de grandes entreprises mexicaines et étrangères, ainsi que des cabinets de professions libérales. Ce retournement de la dynamique démo-économique locale a inauguré une longue période de détérioration du quartier, le départ des résidents et la dégradation du cadre de vie s'accélérant après le séisme de $1985^{30}$. Au total, la présence d'un important tissu de magasins de gros

25 - R. P. Roman Reyes, «Participación familiar en micronegocios en México», Tesis de doctorado, El Colegio de México, 2006.

26 - Ce phénomène doit être relié d'une part à la forte concentration spatiale des vendeurs de textiles, vêtements et chaussures, qui confère aux organisations des marchands une fonction de négociation relative à la localisation et au nombre de points de vente, et d'autre part à la nature des biens vendus - à fort contenu en contrebande et en produits contrefaits, etc. - qui rend nécessaire la création d'un rapport de forces collectif envers les autorités.

27 - G. Garza (coord.), La organización espacial del sector servicios en México, México, El Colegio de México, 2006

28 - Pour les cas des villes de Guadalajara, Mexico et de Puebla, on se reportera à P. Melé, Patrimoine et action publique au centre des villes mexicaines, Paris, Presse de la Sorbonne nouvelle - IHEAL, 1998.

29 - C. Alba et P. Labazée, "Mutation des activités et réhabilitation du centre historique de Mexico. Les politiques urbaines face aux pratiques d'acteurs », Séminaire Réinvestir le centre. Politiques de requalification, transformations urbaines et pratiques citadines dans les grandes villes d'Amérique latine, Paris, CREDAL - CRNS - IRD - Université Paris-III, 2006.

Le Porfiriat est la période marquée par le régime dictatorial du général Porfirio Díaz (1876-1911).

30 - La baisse des résidents dans le centre a été de 34 \% dans les deux dernières décennies, et de plus des deux tiers des années 1950 à 2000. 
et de demi-gros, fournisseur majeur des petits opérateurs - y compris à l'échelon national - et gigantesque aimant commercial dans cette ville de 20 millions d'habitants, et celle d'un système de transport dense, rapide et économique - fin 2006, un billet de métro coûtait 2 pesos soit 0,14 euros - sont les deux facteurs expliquant la localisation préférentielle des ambulants dans un centre où transitent chaque jour entre 1 et 3 millions de personnes ${ }^{31}$.

Il n'existe aucune donnée officielle sur le nombre de points de vente de rue dans la capitale du pays. Le gouvernement du District fédéral ne dispose d'aucun inventaire actualisé, ses estimations actuelles restant fondées sur un dénombrement mené en 1995 en vue de l'octroi de permis provisoires accordés aux ambulants en fin d'année (romerías). Selon les estimations de la chambre de commerce de la ville de Mexico, le nombre des vendeurs de rue serait passé de 180000 en 1994 à 250000 en 1998, 299000 en 2001 et 530000 en 2005 - soit, à cette dernière date, 28 \% environ du total des ambulants du Mexique. Lors des fêtes de fin d'année, pendant lesquelles des autorisations de vente de rue sont délivrées par les autorités locales, le nombre d'ambulants exerçant dans le centre historique et les zones marchandes adjacentes Tepito, Lagunilla, la Merced - aurait atteint 45000 en 2005, contre 13000 en $1996^{32}$. En 2006, selon l'association des commerçants établis du centre historique, ils auraient été près de 60000 . Une enquête menée en 2006 dans une partie du centre auprès de plus de 500 opérateurs ${ }^{33}$ permet de distinguer trois types différents d'intervenants. En premier lieu, la majeure partie des points de vente repose exclusivement sur une base familiale - couples sans ou avec enfants, associations de frères, femmes chefs de ménage. En second lieu, certains propriétaires gèrent un ou plusieurs points de vente en embauchant des salariés dont les rémunérations varient de 700 à 1000 pesos par semaine - entre 50 et 70 euros environ. Enfin, divers commerces établis disposent de points de vente de rue; ces postes dédoublés (puestos desdoblados) visent, selon le cas, à protéger l'établissement principal des nuisances occasionnées par les ambulants, ou à engranger les bénéfices de l'informalité.

\section{Vendeurs ambulants et petits ateliers: des capacités différenciées de survie dans le contexte d'ouverture}

Une rapide comparaison des spécificités respectives du commerce ambulant et des micro-unités de production permet de comprendre pourquoi celui-là s'est considérablement développé dès la fin de l'ère de l'industrialisation par substitution des importations, tandis que le poids relatif des ateliers tendait à se tasser. Au-delà de similitudes de caractéristiques touchant à la création et à l'organisation interne des commerces et des micro-industries - faibles barrières à l'entrée, peu de capital et de technologies, division limitée du travail, forte intensité de main-d'œuvre, flexibilité du volume et des statuts

31 - V. Islas, S. Hernández et S. Blancas, El transporte en la región de México. Vol.1, Diagnóstico general, Sanfadila, SCT, Publicación técnica $\mathrm{n}^{\circ} 232,2004$.

32 - Voir El Economista, 22 mai 2006.

33 - Il s'agit de la zone de Mixcalco, spécialisée dans la vente de vêtements. Quelques résultats de cette enquête ont été exposés dans C. Alba, A. Escobedo, P. Labazée et J. Parra, Mutaciones de actividades en el Centro histórico de la ciudad de México, Miércoles científicos, México, IFAL, 28 juin 2006. 
d'emploi, fragilité et durée de vie limitée d'une proportion élevée d'unités -, il apparaît que le processus d'ouverture a été défavorable aux petits ateliers, ceux-ci ayant dû affronter la concurrence de produits importés à faible contenu technologique et à prix bas, notamment d'origine asiatique ${ }^{34}$, tandis que l'ouverture aux échanges mondiaux contribuait à créer des centaines de milliers d'emplois dans la distribution de biens importés, de façon légale ou non, sur le territoire national. La substitution d'emplois productifs par des activités marchandes a pris des formes diverses qui restent, pour une bonne part, à découvrir et à mesurer. Dans divers pôles du vêtement du pays, par exemple à Moroleón et Uriangato (Guanajuato), nombre de patrons de petits ateliers se sont reconvertis dans la revente de textiles et vêtements de contrebande dans les années 1990, ou ont migré aux États-Unis ${ }^{35}$. À la même époque, d'importants marchés de gros et demi-gros de textiles de contrebande et de contrefaçon apparaissaient dans l'État de Mexico, à Tlalnepantla, Pantaco, San Juan de Aragón par exemple, en vue d'approvisionner les circuits de revente de la capitale du pays. À León, ville dont provient la moitié de la production nationale de chaussures, un grand nombre de fabriques ont fermé leurs portes, et les ateliers se sont repliés sur des activités mixtes de microproduction et de commercialisation de produits asiatiques.

La nature des liens à l'espace urbain, ainsi que le degré et le type de cohésion sociale, constituent d'autres différences opposant les micro-industries et les petits commerces. Ainsi, le centre de la ville de Mexico accueillait de nombreux ateliers familiaux et fabriques de vêtements jusqu'au tremblement de terre de 1985, date à laquelle l'illégalité de ces implantations et les dures conditions de travail dans ces établissements ont été mises à jour ${ }^{36}$. Depuis lors, les activités productives ont fermé ou se sont déplacées vers les quartiers populaires de la périphérie urbaine, voire dans les zones rurales de l'État de Mexico, poursuivant ainsi leur activité hors des obligations imposées par la loi fédérale du travail, la sécurité sociale et l'administration fiscale. En revanche, la survie et l'expansion du petit commerce dans le centre s'expliquent par leur capacité à avoir négocié, de façon collective et avec les autorités locales, la reconduction de la tolérance dont ils bénéficient pour exploiter les lieux les mieux desservis et achalandés : la croissance du nombre des ambulants aux abords du Zócalo est le résultat combiné du dense réseau de lignes de métro desservant le centre-ville, et des pactes passés entre les pouvoirs politiques et les associations de vendeurs ${ }^{37}$. De même, la densité de l'ambulantage selon les

34 - Vêtements, chaussures et autres produits en cuir, meubles et jouets par exemple. Voir E. Dussel, M. Piore et C. Duran, Pensar globalmente y actuar regionalmente. Hacia un nuevo paradigma industrial para el Siglo XXI, México, UNAM - Fundación F. Ebert-Ed. Jus, 1997

35 Voir M. Estrada et P. Labazée («Transformations productives dans l'État de Guanajuato (Mexique). Entrepreneurs locaux et mondialisation», Revue Tiers Monde, $n^{\circ} 181,2005$, p. 119-139) signalant les diverses adaptations des quelque 1350 micro-ateliers locaux face à l'irruption de produits de maille provenant d'Asie.

36 - F. Wilson, De la casa al taller, Zamora, El Colegio de Michoacán, 1990; José A. Alonso, Maquila domiciliaria y subcontratación en México en la era de globalización neoliberal, México, Plaza y Janés, 2002.

37 - Ces accords, qui contreviennent à diverses réglementations, sont parfois interprétés comme le produit d'une normativité extra-légale, au sens de De Soto; en fait, on peut en douter dans la mesure où ces accommodements reconduisent, de façon systématique, les dépendances des vendeurs envers les pouvoirs politiques, notamment la précarité du droit à occuper les rues du centre-ville (C. Alba et P. Labazée, "Acteurs économiques et enjeux politiques dans le centre historique de Mexico. Le commerce ambulant au cour de la controverse post-électorale», Problèmes d'Amérique latine, à paraître). 
rues et les carrefours est proportionnelle au transit piétonnier, générant de fortes variations du coût de location des places fixé par les responsables d'associations de vendeurs et, partant, de nombreux conflits touchant au contrôle politique de celles-ci - conflits qui dégénèrent parfois jusqu'au crime ${ }^{38}$. Les compétitions entre associations n'interdisent pas certaines coopérations entre elles, notamment lorsque des opérations de police sont engagées afin de faire évacuer les rues et de saisir les marchandises des ambulants : chaque organisation dispose d'agents dotés de talkies-walkies et de codes permettant d'aviser les vendeurs de l'imminence d'une action répressive. Dans de nombreux cas, les associations sont préparées à s'opposer à la police, y compris par la violence ${ }^{39}$. Ainsi, tandis que la déconcentration spatiale des petits ateliers anciennement implantés dans le centre leur permettait d'échapper aux contraintes réglementaires et de reconduire leur contrôle sur une main-d'œuvre dispersée, parfois d'origine rurale, les ambulants tiraient de leur concentration un avantage en termes de visibilité, de préservation d'un droit d'usage sur l'espace public, d'organisation et de capacité de mobilisation.

L'absence presque totale de représentation professionnelle des petits producteurs mexicains a facilité la mise en ouvre accélérée des politiques nationales d'ouverture aux concurrences mondiales, à la différence d'autres pays émergents où la libéralisation de secteurs-clés pour l'emploi a été moins abrupte ${ }^{40}$. Pour diverses raisons tenant notamment à l'histoire politique des milieux d'entrepreneurs mexicains, la participation des petits patrons aux structures de représentation professionnelle est en effet restée marginale ${ }^{41}$, l'individualisme du milieu poussant de plus à des stratégies personnelles d'évitement des contraintes publiques. À l'inverse, les ambulants ont très tôt établi leurs organes d'intermédiation et de négociation avec les pouvoirs politiques, administratifs et répressifs. Au cours des soixante-dix années de gouvernement priiste du District fédéral, les organisations de vendeurs de rue ont été intégrées au sein de la Confederación Nacional de Organizaciones Populares (CNOP), structure corporatiste regroupant tous les secteurs urbains ne relevant pas des organisations ouvrières (CTM) et paysannes (CNC). La représentation des ambulants était alors concentrée dans les mains d'un petit nombre de lideres,

38 - Une responsable (lideresa) de premier plan des ambulants du District fédéral, Alejandra Barrios - à la tête de la Asociación Cívica Legítima Comercial depuis vingt-cinq ans - a été incarcérée pendant plus de deux ans, soupçonnée d'avoir organisé en 2003 le meurtre de Jorge Ramírez Espíndola, époux de la lideresa María Rosete, l'une de ses concurrentes politiques dans le milieu des vendeurs de rue. Libérée en décembre 2005 faute de preuves, Alejandra Barrios s'intégra à la CROC, la puissante confédération d'organisations populaires du Parti révolutionnaire institutionnel (PRI), afin - selon ses dires - de faire établir une loi en faveur des travailleurs non salariés, tandis que sa fille Marcela Jiménez Barrios apportait son soutien au candidat du Parti de la révolution démocratique (PRD) à la présidence de la République, Andrés Manuel López Obrador.

39 - Ainsi, le 9 décembre 2006, un affrontement violent opposa les commerçants informels armés de bâtons, de tubes et de pierres, et les policiers de la Secretaría de Seguridad Pública, faisant un bilan de huit policiers blessés et six interpellations, outre divers dégâts matériels. Cette altercation faisait suite à une nouvelle forme d'action répressive, consistant à immobiliser les véhicules mal garés dans le centre, ceux-ci appartenant souvent aux vendeurs ou à leurs fournisseurs. Un autre affrontement violent eut lieu début janvier 2007, portant sur le devenir d'une rue du quartier Casas Alemán où les vendeurs comptaient implanter un tianguis spécialisé dans le jouet.

40 - Y.-A. Fauré, L. Kennedy et P. Labazée, op. cit., 2005.

41 - C. Alba, Los empresarios y la democracia en México, México, El Colegio de México, mimeo., 2006. 
notamment de femmes ayant créé de puissantes associations liées au Parti révolutionnaire institutionnel (PRI) ${ }^{42}$. L'arrivée du Parti de la révolution démocratique (PRD) à la tête du District fédéral en 1997 a profondément modifié le paysage des organisations de vendeurs et des lideres qui les dirigent. Les compétitions entre PRI et PRD pour le contrôle politique de l'ambulantage ont conduit d'une part à la multiplication du nombre des organisations et à leur fragmentation ${ }^{43}$, à l'apparition de conflits et tensions entre elles, d'autre part à de nouveaux rapports - non exclusivement corporatistes, mais le plus souvent marqués par des relations clientélistes - entre le PRD et les associations qu'il contrôle ${ }^{44}$.

La vulnérabilité juridique est une caractéristique commune aux deux milieux de petits opérateurs, mais ses modalités, son intensité et ses effets sont notablement différents. Les ateliers de production ne respectent pas les obligations fiscales ni la réglementation du travail, mais échappent aisément aux contrôles administratifs en raison de leur atomisation et de leur mobilité - l'administration fiscale n'ayant d'ailleurs pas la capacité de les contrôler à la périphérie des villes, et moins encore dans les campagnes. Pour leur part, les agents chargés de la protection sociale n'interviennent qu'en cas de plaintes, relativement peu fréquentes, déposées par les employés. Les petits commerçants de rue, quant à eux, sont juridiquement plus exposés, tant par leur visibilité que parce qu'ils transgressent une plus vaste gamme de dispositions légales : lois sur le transit véhiculaire et le blocage des rues, bando [décret, ordonnance] interdisant le commerce ambulant dans une partie du centre historique $^{45}$, lois fiscales concernant les bénéfices et l'impôt sur la valeur ajoutée (IVA), réglementations touchant au paiement d'impôts et de taxes sur les produits importés, lois de protection des droits de propriété sur les marques déposées, lois sanitaires sur la préparation et la vente d'aliments, branchements sauvages sur le réseau électrique ${ }^{46}$, loi de protection des monuments historiques sur lesquels sont attachés les cordages retenant les bâches, etc. À la différence de la situation des patrons

42 - Sur l'histoire des organisations de vendeurs ambulants de la ville de Mexico, on se reportera à J. Cross, Informal Politics. Street Vendors and the State in Mexico City, Stanford, Stanford University Press, 1998.

43 - Plusieurs dizaines d'associations de vendeurs sont ainsi nées à partir de la fin des années 1990, dont les responsables sont affiliés ou soutiennent le PRD de la capitale; la plupart sont de faible dimension, à l'exception de la Unión de Comerciantes de los hijos de Tepito, dirigée par María Rosette. Le PRI continue de contrôler un petit nombre de grandes organisations de vendeurs ambulants, telle celle d'Alejandra Barrios, évoquée plus haut.

44 - C. Alba et P. Labazée, art. cit., à paraître.

45 - Émis en 1993 par l'assemblée législative du District fédéral, ce bando visait à éliminer l'activité marchande de rue dans le périmètreA, dans le cadre d'une réinstallation - ayant largement échoué - des vendeurs au sein de places commerciales. Cette interdiction, toujours d'actualité malgré l'abandon des politiques de réinstallation des ambulants dès 1996, est au fondement de la précarité des petits opérateurs et, partant, de la dépendance de ceux-ci envers leurs leaders et les partis politiques (A. Escobedo, «Economía y política ambulantes. El comercio popular en las calles del centro histórico de la ciudad de México", México, tesis, El Colegio de México, 2006).

46 - La Direction générale de la protection civile estime à plus d'un million le nombre de diablitos - terme désignant ces branchements illégaux - greffés sur les installations électriques. Selon cette administration, le prélèvement illégal de courant est une pratique généralisée dans les quartiers périphériques et parmi les vendeurs ambulants; mais elle n'est pas rare dans les quartiers aisés, ni de la part des commerçants établis. Récemment, la compagnie Luz y Fuerza del Centro a estimé que la perte sur facturation engendrée par les diablitos était de l'ordre de $20 \%$ (Reforma, 11 décembre 2006). 
d'ateliers, l'extrême vulnérabilité juridique des petits marchands est un puissant vecteur de la reproduction de dépendances multiples, envers les lideres et leurs organisations chargées de négocier les tolérances pour l'usage de l'espace public, envers les autorités locales et fédérales compétentes en matière de fiscalité et de fraude sur les marchandises vendues ${ }^{47}$, envers les instances législatives divisées - comme on le verra plus loin - sur le type de loi répressive à promouvoir sur la contrebande, comme envers les délégations disposant d'un pouvoir régalien de reconnaissance des lideres, des associations de vendeurs et de leur aire d'implantation via l'octroi de permis provisoires.

Les rapports entre commerçants informels et autorités publiques transcendent finalement les clivages entre les partis, renvoyant à un mélange de clientélisme et de corporatisme du système politique mexicain qui se perpétue malgré le virage libéral. Ces rapports recoupent divers enjeux, dont la redistribution informelle des richesses, et engendrent à l'occasion des violences liées aux compétitions entre les organisations pour le contrôle des rues et des rentes liées à l'ambulantage.

\section{L'informel et l'illégal depuis l'ouverture économique : l'insertion de l'économie populaire dans la mondialisation}

Au Mexique, la progression du commerce ambulant au cours des vingt dernières années a coïncidé avec une croissance significative des ventes de marchandises de contrebande et de contrefaçon sur le marché national, portant sur divers biens de consommation courante antérieurement élaborés dans le pays. Même si on ne dispose d'aucune information statistique homogène sur le poids des activités illicites ${ }^{48}$ par type de biens, le croisement de diverses sources permet d'identifier les produits concentrant l'essentiel des flux, parmi lesquels figurent les vêtements, textiles, chaussures, jouets, céramique, verrerie, appareils électriques et électroniques, musique et films, softwares, parfums, huiles, poissons, produits alimentaires ${ }^{49}$. Le cas des vêtements est exemplaire, les sources publiques et privées convergeant pour estimer que plus de la moitié de la consommation nationale revenait, en 2000, aux ventes de biens commercialisés illégalement sur le territoire ${ }^{50}$. Des méthodologies de même nature conduisent à évaluer les entrées illégales de jouets à 800 millions de dollars annuels, tandis que plus de $70 \%$ des chaussures

47 - Il faut noter que les délits touchant à la contrebande relèvent des pouvoirs fédéraux, de sorte que les autorités locales se déclarent non compétentes pour intervenir.

48 - Sur les définitions des divers segments de l'économie non enregistrée mexicaine, on se reportera à F. Roubaud (L'économie informelle au Mexique. De la sphère domestique à la dynamique macro-économique, Paris, Karthala-Orstom, 1994), en notant néanmoins que la période contemporaine rend de plus en plus incertaine l'opposition entre les unités du secteur informel et celles du secteur illégal.

49 - À partir du croisement de diverses sources, dont l'Administration générale des douanes (AGA), la Chambre nationale de commerce (Canaco), la Confédération des chambres d'industrie (Concamin); en ce qui concerne les textiles et vêtements, les données chiffrées relatives à la contrebande sont notamment issues des travaux de E. Dussel et L. Dong (op. cit., 2004), des rapports émis par le cabinet Kurt Salmon Associates pour le compte de la Banque nationale du commerce extérieur, et des informations fournies par les Chambres nationales de l'industrie textile et de la confection (Canaintex et Canainvest).

50 - Soit, selon les estimations, entre 8,5 et 9,4 milliards de dollars sur un total de consommation apparente de 16,3 milliards; voir Kurt Salmon y Asociados (KSA), El mercado mexicano de ropa, México, KSA-Canainvest, 2002. 
importées, soit plus de 23 millions de paires, proviennent de la contrebande "technique ${ }^{51}$. Les flux de biens de fraude, de même que ceux portant sur les substances illicites, existaient certes aux $\mathrm{XIX}^{\mathrm{e}}$ et $\mathrm{Xx}^{\mathrm{e}}$ siècles, soit bien avant les politiques $\mathrm{d}^{\prime}$ ouverture commerciale ${ }^{52}$; ils étaient alors stimulés par les différentiels de prix issus des politiques de protection des industries nationales. En revanche, l'envolée contemporaine de la contrebande dans un contexte de large démantèlement des barrières à l'importation semble à première vue paradoxale. En fait, la contrebande a progressé sur les marchandises restant soumises à des droits de douane élevés, ou à des quotas compensatoires lorsqu'il est démontré qu'elles sont introduites dans des conditions de discrimination de prix (dumping) au sens de la loi du commerce extérieur ${ }^{53}$ - ce qui est le cas des textiles, vêtements et chaussures en provenance de Chine. Mais les flux de contrebande concernent aussi divers biens sur lesquels les droits de douane sont modestes, dès lors que l'ampleur des volumes importés et le non-paiement des diverses taxes et de l'impôt sur la valeur ajoutée (IVA) laissent des marges substantielles aux acteurs de ces filières - situation désormais fréquente, puisque les nouvelles techniques contrebandières ont eu pour effet de réduire les coûts relatifs à l'entrée en fraude des biens sur le territoire.

Ces causes n'expliquent pas à elles seules la progression de la fraude qui s'inscrit dans un désajustement général entre, d'un côté, l'accélération et la diversification des échanges mondiaux et, de l'autre, un corpus ancien de lois, de règlements et d'institutions chargés de les encadrer. Ainsi, les services douaniers sont-ils sous-équipés en effectifs pour contrôler les importateurs dont le nombre a considérablement progressé dans les dernières années ${ }^{54}$, et en experts spécialisés dans la détection de fraudes sur les produits les plus sensibles ${ }^{55}$. Par ailleurs, la coopération douanière est des plus réduites avec les États-Unis, pays par lequel transitent $70 \%$ des importations mexicaines, la détection de fraudes n'étant pas une priorité du point de vue nordaméricain $^{56}$, ainsi qu'avec les pays d'où les marchandises proviennent. En outre, les possibilités légales d'actions anti-dumping prévues par la Ley de Comercio Exterior sont particulièrement lentes et coûteuses, l'administration de la preuve revenant aux

51 - Ensemble des modalités visant à faire entrer les biens par de fausses déclarations relatives à leur volume, leur valeur, leur origine ou leur nature.

52 - W. L. Bernecker, Contrabando. Ilegalidad y corrupción en el México del Siglo XIX, México, Universidad Iberoamericana, 1994 ; J. Rivelois, «Drogue, corruption et métamorphoses politiques (application à une comparaison Mexique-Brésil)», Revue Tiers Monde, t. XL, n²158, 1999.

53 - N. Simón, «Prácticas desleales e ilegales de comercio internacional en las industrias de textiles y prendas de vestir en México», in I. Rueda Peiros, N. Simón et M. L. González (coord.), La industría de la confección en México y China ante la globalización, México, UNAM-Porrúa, 2004.

54 - Pour la seule année 2004, plus de 6000 demandes d'inscription au Padrón de Importadores ont été déposées, dont environ $60 \%$ ont été rejetées pour non-respect des obligations fiscales ou défaut de conformité envers l'enregistrement au Registre des contribuables, voir Administración de Padrón de Importadores, México, SHCP-SAT, 2005.

55 - En juillet 2005, l'administration des douanes disposait ainsi de onze experts spécialistes dans l'importation de chaussures pour l'ensemble des quarante-huit douanes du pays, ceux-ci étant de plus chargés de l'inspection d'autres biens, notamment les vêtements pour lesquels il n'existait aucun expert.

56 - Les informations sur les exportateurs nord-américains sont par exemple confidentielles, et le montant des flux exportés n'est pas directement transmis par la douane, mais par le bureau de recensements (Census Bureau) des États-Unis, dont les temps de réponse sont lents, voir Entretien avec J. Guzmán Montalvo, directeur AGA, Revista Fortuna, Negocios y Finanzas, juillet 2005. 
entreprises plaignantes. Elles restent hors de portée des micro, petites et moyennes entreprises, qui constituent l'essentiel du secteur productif national. Elles ne sont mobilisées que par de grands groupes industriels et les chambres d'industrie ${ }^{57}$. Dans un contexte de croissance des flux et du nombre des opérateurs de contrebande, divers observateurs contestent aussi la fiabilité des délivrances de certificats (amparos) autorisant l'entrée de marchandises contre des cautions modestes, leur usage facilitant la fraude, notamment sur les importations temporaires ${ }^{58}$. L'un des débats manifestant le plus clairement le désajustement entre le cadre juridique et institutionnel et la réalité des échanges contemporains concerne l'engagement de poursuites contre les acteurs de la contrebande et du piratage. Le délit de contrebande n'étant pas inscrit dans la loi fédérale contre la délinquance organisée, il ne pouvait être poursuivi qu'à partir d'une plainte déposée au préalable auprès de la Secretaría de Hacienda y Crédito Público, non sur la base d'opérations ou d'enquêtes de police. Plus de trois ans de discussions au Congrès de l'Union ont été nécessaires pour modifier certains aspects de la loi, tant les enjeux politiques et financiers ont opposé les divers groupes de pression présents dans l'enceinte parlementaire - chambres d'industries, groupes industriels, importateurs et agents des douanes ${ }^{59}$. Toutefois, fin 2006, aucune application des nouvelles dispositions n'était perceptible, tant dans le domaine de la contrebande que dans celui de la contrefaçon. L'articulation de l'administration des douanes aux principaux réseaux organisant la contrebande est sans doute un facteur puissant de préservation de ces divers désajustements, malgré la récurrence des sanctions - 2550 agents sanctionnés ou licenciés entre 2001 et 2005, forte accélération de la rotation des personnels de l'inspection fiscale et douanière à cette même période - envers les douaniers ${ }^{60}$.

Selon les chambres d'industrie textile et de confection, l'essor de la contrebande est l'une des deux causes principales du déclin de la production nationale, l'autre tenant à la chute continue des crédits bancaires ${ }^{61}$ octroyés à un secteur d'activité désormais considéré comme à haut risque. Entre 2000 et fin 2005, le nombre d'établissements de confection est passé de 14019 à 11 017, l'emploi passant de 640000 à 399000 travailleurs. Pour la Cámara Nacional de la Industria del Vestido (Canainvest), le gouvernement est en train d'abandonner la bataille de la contrebande de textiles, les saisies réalisées en 2005 représentant à peine $10 \%$ de celles réalisées l'année antérieure $^{62}$. Les principales raisons avancées par les entrepreneurs mexicains concernent l'absence d'investigations "d'intelligence», c'est-à-dire de recoupements systématiques d'informations, la sophistication croissante des techniques de fraude

57 - N. Simón, art. cit., 2004.

58 - Voir Senadora Dulce M. Sauri Riancho, «Propuesta para referirse al problema del contrabando", México, Gaceta parlamentaria, 4 septembre 2003.

59 - Entretien avec Roberto Castañeda, responsable de Alianza contra la Piratería, Mexico, 3 juin 2005.

60 - Selon les informations de l'administration douanière, 76 dossiers de contrebande dite "technique» ont été instruits entre 2001 et 2005, le tiers des personnes inculpées faisant partie de cette même administration.

61 - Les crédits bancaires à l'industrie textile et de confection sont passés de 26,6 milliards de pesos en 1998 à 17,8 milliards en 2003, voir La Industria Textil y del Vestido en México, Estadísticas sectoriales, Aguascalientes, INEGI, diverses dates.

62 - Soit 6800 rouleaux de tissus de $200 \mathrm{~m}^{2}$ en 2005, contre 73300 en 2004. 
- manipulation des rubriques douanières, falsification des documents administratifs, emploi de l'Internet pour passer et suivre les commandes -, la multiplication des importateurs et d'entreprises-écran appelées empresas portafolios, qui rendent difficile le suivi des circuits de contrebande ${ }^{63}$, le déplacement des priorités répressives sur d'autres produits, notamment les chaussures et les médicaments ${ }^{64}$.

L'une des caractéristiques de la contrebande contemporaine réside dans l'extrême diversité des acteurs, des circuits et des techniques d'approvisionnement du marché national. Notons d'abord que la vente ambulante n'est qu'une modalité de distribution parmi d'autres des biens entrés illégalement sur le territoire, bien qu'elle soit la plus connue - parce que la plus visible - de la part des consommateurs, des pouvoirs publics et des entrepreneurs du secteur formel ${ }^{65}$. Nombre des plus grands groupes de distribution, supermarchés et chaînes de magasins spécialisés, qui représenteraient environ 55 \% du marché national du vêtement ${ }^{66}$, ont en effet eu recours à des importations illégales - cas de Gigante, Liverpool, Zara-Inditex ${ }^{67}$ pour ne mentionner ici que des opérateurs ayant fait l'objet de pénalisations -, la triangulation étant la démarche la plus courante pour l'entrée en contrebande de volumes importants ${ }^{68}$ exclusivement revendus dans les espaces commerciaux de ces enseignes. La surface financière de ces intervenants, et le soutien reçu à l'occasion des États hébergeant les sociétés mères, les rendent peu vulnérables aux enquêtes douanières ${ }^{69}$. Une partie de l'industrie maquiladora d'exportation (IME) participe aussi aux circuits de contrebande, notamment sur les tissus, via le détournement des règles d'importation temporaire inscrites aux deux programmes officiels Pitex et Maquila. L'ampleur des flux reversés sur le marché national a du reste conduit l'administration générale des douanes (AGA) à renforcer le dispositif de contrôle des entreprises bénéficiaires, en créant la figure de l'entreprise "certifiée» afin d'éliminer les établissements sans activité visible ou ceux dont l'existence légale est

63 - Entretiens avec Antonio Kuri, président de la Canainvest, 29 mai 2006, et avec Roberto Castañeda, 4 janvier 2006.

64 - Entretien avec Mauricio Montes de Oca Durán, responsable de la Unidad Especial de Investigación de Delitos fiscales y Financieros de la Procuraduría General de la República, 13 mai 2006.

65 - Cette modalité de diffusion des biens de contrebande a ainsi été mentionnée par l'ensemble des industriels du secteur textile et de la confection interrogés au cours de l'année 2005 dans le District fédéral et l'État de Mexico.

66 - Kurt Salmon y Asociados (KSA), op. cit., 2002.

67 - Groupe espagnol de première importance, Inditex faisait transiter par l'Espagne des vêtements fabriqués en Asie, puis les réexportait vers le Mexique après ré-étiquetage. Le paiement des droits de douane s'opérait selon le barème préférentiel prévu au Traité de libre commerce avec l'Union européenne, soit $5 \%$ - contre $355 \%$ de droits pour les produits provenant de Chine.

68 - La triangulation consiste à faire transiter les produits d'origine asiatique, soumis à des droits élevés, par un pays tiers - principalement les États-Unis - qui les réexporte après modification des documents d'origine et des étiquettes, afin qu'ils bénéficient des avantages douaniers prévus par les accords de libre-échange. Panamá est un autre point de transit des marchandises triangulées et dessert le marché mexicain par le port de Manzanillo.

69 - Voir à ce propos les récentes déclarations de Santiago Pasos, président de la Chambre nationale de l'industrie de la chaussure, soulignant que «les grands supermarchés comme Wal-Mart et Gigante, tous ceux-là, ont une certaine immunité pour le volume de leurs importations, dans le sens où ils ont le feu vert, la loi les protège, mais les révisions faites après des petits importateurs semblent incongrues et risibles, alors qu'on ne révise pas les grands importateurs", cité dans Fortuna, Negocios y Finanzas, juillet 2005. 
mal assurée $e^{70}$. Une fois transformés par les industries ou par leurs sous-traitants, les textiles sont cédés auprès d'établissements de commerce formels, ou auprès de grossistes fournissant les petits opérateurs de rue ou de tianguis. Ces deux premières modalités de la contrebande attestent du caractère hâtif des approches visant à faire du commerce illégal un segment spécialisé des activités informelles : de fait, les entreprises enregistrées sont bien à l'origine de flux de contrebande, dont une partie approvisionne les milliers de micro-opérateurs de rue. La fraude passe enfin par de multiples réseaux d'importateurs manipulant les déclarations de quantités, les certificats d'origine ${ }^{71}$ et de code douanier, opérations supposant l'appui des déclarants en douane patentés - environ mille au Mexique -, nombre d'entre eux agissant comme négociateurs informels entre les intérêts des importateurs et ceux des agents des douanes ${ }^{72}$. Si ces importateurs sont d'origines très diverses, il faut mentionner le rôle croissant pris depuis quelques années par les commerçants coréens et chinois, dont les relais s'étendent tant vers l'Amérique centrale où une partie des biens est revendue, que dans certaines régions-clés des États-Unis servant de lieux de transit. Les magasins de gros, demi-gros et détail de ces opérateurs se sont multipliés à Tepito depuis 1997, et plus récemment dans la partie nord du centre historique, où leurs achats immobiliers ont fortement progressé; ils sont aussi basés à Guadalajara, Monterrey et dans la plupart des grandes villes frontalières du Nord du pays, ainsi que dans certaines villes et bourgs du centre du pays jouant un rôle important dans la redistribution nationale de biens de fraude ${ }^{73}$. Pour la Canainvest, les réseaux émanant d'une communauté coréenne en croissance seraient devenus les principaux vecteurs de la contrebande nationale. Quoi qu'il en soit, les circuits asiatiques constituent d'importantes sources d'approvisionnement pour les ambulants, dans la mesure où ils disposent de boutiques et de dépôts à proximité des principaux centres de distribution finale, la revente pouvant de plus faire l'objet de crédits à 30 ou 60 jours. On mentionnera enfin que la contrebande par franchissement clandestin des frontières - appelée contrabando bronco, par opposition aux techniques plus élaborées - existe certes toujours, mais sa part est devenue marginale dans l'ensemble des flux : elle ne concerne que les petits commerçants des zones frontalières du pays, la région de Mérida ${ }^{74}$, ainsi que quelques opérateurs de la ville de Mexico, parfois liés aux lideres qui encadrent les associations de vendeurs.

70 - Voir AGA, 2002, Información para solicitar el dictamen favorable del CNIME para la inscripción en el Registro de Empresas Certificadas bajo el Rubro D de la RCGMCE, México, Consejo Nacional de la Industria Maquiladora de Exportación.

71 - Ces certificats sont obligatoires pour toute importation minimale de textiles, vêtements et chaussures provenant notamment de Corée du Sud, Hong Kong, Inde, Indonésie, Malaisie, Pakistan, Singapour, Thaïlande, Chine, et d'autres pays non membres de l'OMC.

72 - Entre 2002 et 2005, quatre-vingt-seize déclarants ont ainsi été soumis à des enquêtes pénales pour leur participation à des opérations de contrebande et de corruption, y compris à de faux programmes de maquilas.

73 - Par exemple à Uriangato (État de Guanajuato), où fut arrêté Park Kang Hysoun, l'un des membres éminents de la communauté résidant dans ce bourg, considéré comme l'un des plus importants distributeurs nationaux de vêtements de contrebande et de contrefaçon.

74 - J. Rivelois, "La défense des espaces urbains centraux par les acteurs de la marge», Séminaire Réinvestir le centre. Politiques de requalification, transformations urbaines et pratiques citadines dans les grandes villes d'Amérique latine, Paris, CREDAL-CRNS - IRD-Université Paris-III, 2006. 
Comme formes d'appropriation illégale d'une idée, d'un concept, d'un dessin ou d'une marque en vue de leur reproduction à des fins commerciales, la contrefaçon et le piratage - qui représenteraient, en 2004, environ $7 \%$ des échanges mondiaux $^{75}$ - existent depuis longtemps dans les pays en développement, leur importance dans l'activité économique ayant progressé, par exemple en Chine, au Brésil, en Argentine et au Mexique, à la suite de la troisième révolution scientifique et technologique et parallèlement à la libéralisation des marchés et à la mondialisation des échanges. L'usage d'Internet a facilité l'accès à l'information et à l'achat décentralisé de matériels peu coûteux, capables de dupliquer les programmes informatiques, la musique, les films et les jeux. La diffusion de ces nouveaux modes de reproduction sur les marchés officiels et informels a déverrouillé le marché du piratage de sons et d'images, encore restreint à l'ère des disques vinyle et des enregistrements sur bandes magnétiques. La flexibilisation de la production a aussi favorisé le transfert de responsabilités et de savoir-faire à des sous-traitants disséminés dans l'espace, les grandes compagnies conservant la gestion des marques et des images dont dépend, finalement, la valeur ajoutée de ces biens. Ainsi, le produit industriel s'est converti en support matériel de marques qui constituent le principal vecteur des concurrences sur les marchés finaux ${ }^{76}$. Dans le cas du marché mexicain du vêtement, dans lequel les marques représentent près du tiers des ventes - soit $34 \%$ et $28 \%$ respectivement pour les vêtements féminins et masculins -, il convient de distinguer deux circuits distribuant les marques. Le premier concerne les grandes chaînes de distribution commerciale - K-Mart, Wal-Mart, Carrefour, etc. - vendant des biens de base à prix modérés, sans souci d'exclusivité ${ }^{77}$; ces distributeurs disposent d'une marque générique pour chaque type de produits, qui est apposée de façon systématique aux marchandises réceptionnées, les fournisseurs pouvant ainsi être remplacés à tout moment au profit de concurrents pratiquant des prix plus bas, sans préjudice pour la marque. Le second diffuse les biens de luxe, depuis Louis Vuitton jusqu'à Levi's, dont les labels appartiennent à des groupes européens ou nord-américains réputés. Nombre des marques exclusives se sont implantées au Mexique pour capter la clientèle nationale à hauts revenus et, à l'occasion, bénéficier des coûts de main-d'œuvre faibles; dans ce dernier cas, les concessions de marque à des fabricants locaux donnent lieu au paiement de royalties représentant 10 à $15 \%$ du chiffre d'affaires, en contrepartie de l'exploitation de modèles spécifiques, du transfert de procédés assurant la qualité de la production, et du prestige de l'image relayée par les investissements en marketing. C'est sur ces produits, notamment de confection, que se concentre le piratage par la réplique de dessins, d'étiquettes, de logos ou marques, d'hologrammes et de techniques de coupe et de couture, piratage réalisé par des ateliers asiatiques ou du secteur informel mexicain.

75 - Point sur la lutte contre la contrefaçon. Rapport mondial, 2004, Bruxelles, Organisation mondiale des douanes (OMD), 2006.

76 - N. Klein, No logo. El poder de las marcas, Barcelona, Paidós, 2000.

77 - Divers entretiens avec les fournisseurs de ces chaînes de distribution indiquent en effet qu'une partie des modèles contractuellement approuvés par les grandes surfaces sont distribués via divers autres circuits, y compris par les opérateurs du secteur informel. 
Au Mexique, une dizaine de groupes importants contrôlent l'essentiel de la production et de la commercialisation de la musique et des films piratés - le pays serait le cinquième distributeur mondial de ces produits d'après le Rapport 2004 de l'organisation mondiale des douanes (OMD) paru en 2006. Ils ont créé leurs marques, une trentaine pour chacun, qu'ils utilisent afin de se différencier de leurs concurrents ${ }^{78}$. Ces groupes disposent d'assesseurs chargés des aspects financiers, logistiques et artistiques de l'activité, et réalisent leurs propres productions à partir de la compilation d'œuvres originales. Ils disposent de relais avec d'autres contrefacteurs situés dans et hors du Mexique, par lesquels ils obtiennent les matériels de duplication, les disques vierges et les œuvres les plus récentes. Leurs réseaux de distribution, complexes et labiles, alimentent les tianguis et les marchés populaires à l'échelle du pays et de l'Amérique centrale - où certaines de leurs marques ont été identifiées. Tepito, quartier jouxtant le centre historique de la ville de Mexico, reste sans doute le premier marché mexicain de produits piratés ${ }^{79}$, bien qu'une réelle décentralisation se soit produite dans les dix dernières années au profit de sites de redistribution plus éloignés, situés au Nord du District fédéral, tels que San Felipe de Jesús, San Juan de Aragón, Tlalnepantla, etc. D’autres villes du pays - Guadalajara, Puebla, Toluca, Veracruz, Mérida notamment - disposent de leurs circuits de production et de distribution ${ }^{80}$. Au total, et selon les données de la Motion Picture Association, 75 millions de DVD de films et 110 millions de CD musicaux piratés auraient été vendus au Mexique au cours de l'année $2006^{81}$. Le pays est par ailleurs un important producteur, exportateur et consommateur de programmes informatiques copiés de façon illégale - ils représenteraient $55 \%$ des achats nationaux ${ }^{82}$-, le taux de consommation de ces produits étant de l'ordre de $66 \%$ en Amérique latine, soit supérieur de $31 \%$ à la moyenne mondiale ${ }^{83}$.

La croissance du marché des biens de contrefaçon distribués à Tepito, dans le centre historique de la capitale et dans la plupart des tianguis des grandes villes du Mexique a conduit quelques grandes compagnies et chambres d'industries à créer la Alianza contra la Piratería A.C. ${ }^{84}$, qui fonctionne comme un groupe de pression envers les pouvoirs

78 - Pour les disques et films, on peut ainsi mentionner Producciones Oso, Gallo Record MP3, Producciones Surfín, Producciones A, Producciones Lobo, Jordan, DVD Guerrero, Fast Record, DVD Line, etc.

79 - S. Alarcón, «El tianguis global», in C. Bueno (coord.), Globalización. Una cuestión antropológica, México, CIESAS-Porrúa, 2000.

80 - À Toluca par exemple, un groupe d'opérateurs exploitant le label de Producciones Charly a été récemment démantelé, la police saisissant quelque mille appareils de reproduction. À l'origine, les opérateurs du piratage ont imposé les marques pour conférer une apparence de légalité à leurs produits qui comportaient à l'occasion un avis de protection des droits d'auteurs. Désormais, la marque aurait acquis un rôle distinctif, voire ostentatoire, soulignant la puissance économique des grandes organisations par rapport aux petits concurrents; elle permettrait d'autre part une meilleure délimitation des territoires et des zones d'influence, comme c'est le cas dans le narcotrafic. Par ailleurs, les groupes commercialisant les CD et DVD disposeraient chacun de plusieurs marques afin de protéger leur production en cas de saisie policière.

81 - Voir Reforma, "Presumen piratas su marca», 2 janvier 2007, à partir des déclarations de divers représentants des compagnies de disques.

82 - Voir Gazeta Parlamentaria, "Iniciativa que reforma la ley de la propiedad industrial, del código penal federal y de la ley federal de derechos de autores», 14 décembre 2005.

83 - Business Software Alliance, Informe anual, 2005; consulté le 7 mars 2006 sur : http://www. bsa.org/mexico.

84 - Les principaux participants sont Microsoft, Ruz, Glaxo Smith Line, Philip Morris Int., Tycoon Enterprises, Levi's, Martí, Disney, Exim Licensing Group Mexico, Canainvest, Adidas, Nike. 
publics, et comme un organe de sensibilisation des consommateurs. En 2005 a aussi été fondé l'Instituto para la Propiedad Intelectual y el Comercio Legal (IPPIC), front plus large d'industriels affectés par le piratage et la contrebande, dont l'un des buts est de dénoncer «l'incapacité des autorités fédérales, des États et des localités à développer une stratégie de recherche sur le crime organisé ${ }^{85}$ ». Pour ces entreprises, dont certaines disposent de leur propre système d'information sur les marchés illégaux, les pouvoirs publics ont abandonné l'étude des grands réseaux structurant la fraude - et, partant, les liens qui les articulent à diverses institutions d'État - au profit d'une politique privilégiant la comptabilité des saisies ponctuelles, dont l'effet est quasi nul sur les organisations délictueuses. De même exigent-elles que la récente Commission intersectorielle de lutte contre l'illégalité, créée en 2006, devienne opérationnelle et s'inscrive dans la durée $^{86}$. Enfin, l'IPPIC mène un travail de lobbying pour que tous les opérateurs et bénéficiaires de la contrefaçon et de la contrebande, y compris les consommateurs, soient réprimés sans nécessité d'un dépôt de plainte préalable (por oficio) ${ }^{87}$.

Les rapports de forces entre les lobbies industriels, les réseaux de contrefaçon et de contrebande et les pouvoirs publics sont donc loin d'être stabilisés. Le tournant libéral du Mexique et les déréglementations qui en sont le résultat ont, en effet, affaibli les institutions étatiques de contrôle et de répression de l'illégalité, au moment même où les opportunités d'importations non enregistrées et de piratage se développaient. La mise en place de nouvelles institutions et le vote de nouvelles lois répressives se heurtent ainsi au poids économique des réseaux illégaux, et aux enjeux sociaux et politiques dans lesquels ils sont parvenus à s'insérer.

\section{Conclusion}

De même que dans la plupart des pays latino-américains, les politiques libérales et d'ouverture commerciale au Mexique ont provoqué un fléchissement dans la création de postes de travail dans le secteur formel, tenant d'un côté à l'affaiblissement de filières d'activités anciennes à fort contenu en travail - textile et confection, chaussure, jouets notamment -, et de l'autre aux difficultés d'expansion, au tournant du siècle, de l'industrie maquiladora d'exportation. L'informalisation des activités et des emplois en est l'un des effets les plus manifestes : environ 370000 travailleurs intègrent annuellement le secteur des micro-entreprises, chiffre supérieur aux créations de postes de travail dans les unités du secteur formel. Toutefois, la progression de l'informel mexicain tient principalement à l'essor des petits commerces et des services liés à des «complexes» correspondant à quelques besoins de base des citadins : dans un contexte de contraction des pouvoirs d'achat des citadins, les micro-activités en forte croissance sont celles qui répondent à quatre demandes essentielles - se loger, se nourrir, se vêtir, se déplacer -, à partir de la commercialisation, légale ou non, de biens à faible coût.

85 - Instituto para la Protección de la Propiedad Intelectual y el Comercio Legal (IPPIC), Combatir la ilegalidad y la recuperación del mercado interno, Boletin de Prensa, México, 2006.

86 - Cette commission dépend du ministère de l'Intérieur (Secretaría de Gobernación).

87 - Le 14 décembre 2005, la Chambre des députés a ainsi modifié l'article 214 bis de la loi sur la propriété intellectuelle, en prévoyant l'application d'une amende de 100 salaires minimums (4 800 pesos) pour tout consommateur achetant un bien contrefait en connaissance de cause. 
À l'expansion du commerce informel, exercé notamment sans local fixe, répond symétriquement le déclin de petites activités de production dans les secteurs les plus exposés à la concurrence de biens venus du marché mondial. Tel est le cas des ateliers de confection et de chaussures, dont les patrons ont amorcé au cours des années 1990 de difficiles reconversions, soit en migrant vers les États-Unis, soit en se recyclant vers le petit commerce. L'absence de représentation professionnelle des patrons d'ateliers, les stratégies individuelles de contournement des réglementations, l'enracinement faible des ateliers dans leur espace urbain expliquent qu'aucune réponse collective n'ait été élaborée afin d'endiguer la contraction d'un marché intérieur progressivement conquis par les importations légales et la contrebande. À l'inverse, les vendeurs de rue disposent d'organisations puissantes depuis l'ère du Parti révolutionnaire institutionnel, les concurrences entre partis politiques ayant, dans nombre de villes du pays, ouvert un contexte favorable à la reconduction des tolérances envers les vendeurs ambulants, dont le nombre et la capacité de mobilisation sont désormais des facteurs importants des compétitions électorales.

Si l'essor de l'ambulantage a pour origine une situation caractérisée par la baisse relative du salariat - avec la disparition de ses attributs habituels : protection sociale, garantie d'emploi, rémunération fixe - dans l'emploi au Mexique, et si la concentration du commerce de rue dans les centres des grandes villes a pour fondement la reconduction des rapports de dépendance entre les pouvoirs locaux et les associations de vendeurs, la croissance de la contrebande et la généralisation du piratage ont, pour leur part, joué un rôle majeur dans l'approvisionnement des nouveaux entrants dans le secteur informel et, partant, dans la préservation des revenus des petits opérateurs. L'envolée de la contrebande dans les deux dernières décennies tient d'abord au contournement, par des réseaux d'opérateurs nationaux ou étrangers, de droits compensatoires sur des produits importés à des prix de dumping. Mais elle tient aussi à la généralisation de la contrebande «technique» - triangulation, manipulation des barèmes douaniers, etc. - qui a progressivement supplanté la contrebande physique. Cette mutation des modalités de la fraude a eu pour effet d'abaisser considérablement les coûts d'entrée illégale des biens. La contrefaçon, pour sa part, a permis aux catégories à revenus faibles et intermédiaires d'accéder à des produits difficilement abordables - musique, films, softwares, matériels électroniques, vêtements, chaussures, accessoires de mode, etc. Plus fondamentalement, la contrebande et le piratage prospèrent sur un profond désajustement entre les nouvelles règles de l'échange mondial et le corpus de lois, règlements et institutions répressives conçus à une ère de verrouillage des flux marchands.

La diversité des opérateurs de la contrebande est sans doute l'un des traits marquants de l'ère contemporaine. Certaines grandes chaînes de distribution, diverses industries maquiladoras, nombre d'importateurs certifiés, divers petits entrepreneurs de la fraude participent à ce marché en expansion, la plupart distribuant les produits importés via l'économie populaire - tianguis ou vendeurs de rue. Une telle articulation entre les grands réseaux travaillant aux marges de la légalité et les petits opérateurs du secteur commercial renouvelle en profondeur les débats relatifs à l'interprétation de l'informalité, celle-ci s'entendant comme le dernier maillon - économiquement et politiquement dominé - d'une dynamique libérale sans entraves. 


\section{Bibliographie}

- Alarcón S., «El Tianguis Global», in C. BuENO (coord.), Globalización. Una cuestión antropológica, México, CIESAS-Porrúa, 2000.

- Alba C., «L'industrie maquiladora dans le contexte des transformations régionales", in Y.-A. Fauré, L. Kennedy et P. LABAzée, Productions locales et marché mondial dans les pays émergents. Brésil, Inde, Mexique, Paris, IRD-Karthala, 2005.

- Alba C., Los empresarios y la democracia en México, México, El Colegio de México, mimeo., 2006.

- Alba C. et D. KRUitT, «El significado del sector informal y la microempresa en América latina y en México ", in T. CALvo et B. MÉndez, Micro y pequeña empresa en México. Frente a los retos de la globalización, México, Misceláneas, 1995.

- Alba C.et P. LaBAzÉE, «Mutation des activités et réhabilitation du centre historique de Mexico. Les politiques urbaines face aux pratiques d'acteurs", Séminaire Réinvestir le centre. Politiques de requalification, transformations urbaines et pratiques citadines dans les grandes villes d'Amérique latine, Paris, CREDAL-CRNS-IRDUniversité Paris-III, 2006.

- Alba C. et P. LABAzÉE, "Acteurs économiques et enjeux politiques dans le centre historique de Mexico. Le commerce ambulant au cour de la controverse post-électorale», Problèmes d'Amérique latine, à paraître.

- Alba C., A. Escobedo, P. Labazée et J. Parra, Mutaciones de actividades en el Centro histórico de la ciudad de México, Miércoles científicos, México, IFAL, 28 juin 2006

- Alonso, J. A., Maquila domiciliaria y subcontratación en México en la era de globalización neoliberal, México, Plaza y Janés, 2002

- Álvarez Galván J. L. et C. Tilly, «Trabajadores en el comercio y los servicios en México : trabajo marginal ", in E. de la GARZA et C. Salas (coord.), La situación del trabajo en México, México, Plaza y Valdés, 2006.

- Bernecker W. L, Contrabando. Ilegalidad y corrupción en el México del Siglo XIX, México, Universidad Iberoamericana, 1994.
- Bernecker W. L., «La principal industria del país: contrabando en el México decimonónico", America latina en la historia económica, $\mathrm{n}^{\circ}$ 24, Instituto Mora, 2005.

- Business SoftwARE AluANCE, Informe anual, 2005.

- Cepal-Iheal, Une décennie de développement social en Amérique latine, Santiago du Chili, Nations unies, Livre de la CEPALC n 77, 2004.

- Cour J.-M., Les enjeux de l'urbanisation dans les pays en voie de peuplement, Paris, OCDEClub du Sahel, 1995.

- Cross J., Informal Politics. Street Vendors and the State in Mexico City, Stanford, Stanford University Press, 1998.

- Dussel E., M. Piore et C. Duran, Pensar globalmente y actuar regionalmente. Hacia un nuevo paradigma industrial para el Siglo XXI, México, UNAM-Fundación F. Ebert-Ed. Jus, 1997.

- Dussel E. et L. Dong, Oportunidades y retos económicos de China para México y Centroamérica, México, Naciones Unidas-CEPAL, 2004.

- Escobedo A., «Economía y política ambulantes. El comercio popular en las calles del centro histórico de la ciudad de México", México, Tesis, El Colegio de México, 2006.

- Estrada M. et P. LabazéE, «Transformations productives dans l'État de Guanajuato (Mexique). Entrepreneurs locaux et mondialisation", Revue Tiers Monde, $\mathrm{n}^{\circ} 181$, numéro spécial "Décentralisation et développement local. Un lien à repenser», 2005, p. 119-139.

- Estrada M. et P. LABAzÉE, «Transformaciones productivas en el Estado de Guanajuato. Empresarios locales y globalización", in M. Estrada et P. LABAzÉE (dir.), Globalidad y localidad. Producción, actores, migraciones e identidades, CIESAS - IRD - Casa Chata, 2007.

- Fauré Y.-A. et P. LABAZÉE, Socio-économie des villes africaines, Paris, Karthala, 2002.

- Fauré Y.-A., L. Kennedy et P. Labazée, Productions locales et marché mondial dans les pays émergents. Brésil, Inde, Mexique, Paris, IRD-Karthala, 2005.

- Fondo de CAPITALIZACiÓn E INVERSión DEL SECTOR RURAL (FOCIR), Transformación de la agroindustría 
$y$ del comercio internacional de los alimentos en México, México, AIC-FOCIR, 2005.

- Garza G. (coord.), La organización espacial del sector servicios en México, México, El Colegio de México, 2006.

- Instituto Nacional de Estadística, Geografía e INFORMÁTICA (INEGI), Encuesta Nacional de Micronegocios, Aguascalientes, INEGI, 1994, 2002.

- Instituto Nacional de Estadística, Geografía E Informática (INEGI), Censo Económico, Aguascalientes, INEGI, 1984, 2004.

- Instituto para la Protección de la Propiedad Intelectual y el Comercio Legal (IPPIC), Combatir la ilegalidad y la recuperación del mercado interno, Boletín de Prensa, México, IPPIC, 2006.

- Islas V., S. Hernández et S. Blancas, El transporte en la región de México, Vol. 1, Diagnóstico general, Sanfadila, SCT, Publicación técnica $\mathrm{n}^{\circ} 232,2004$.

- Kelley B., "The Informal Sector and the Macroeconomy : A CGE Approach for Peru», World Development, vol. 22, n 9, 1994.

- Klein N., No logo. El poder de las marcas, Barcelona, Paidós, 2000.

- Kurt Salmon y Asociados (KSA), El mercado mexicano de ropa, México, KSA-Canainvest, 2002.

- Martínez M. de la, "La industria maquiladora en México", in E. de la GARzA et C. SALAS (coord.), La situación del trabajo en México, México, Plaza y Valdés, 2006.

- Marques-Pereira J. et B. Théret, «Régimes politiques, médiations sociales de la régulation et dynamiques macroéconomiques", in Association recherche et régulation, L'année de la régulation. Économie, Institutions, Pouvoirs, $\mathrm{n}^{\circ}$ 5, 2001-2002, Paris, Presses de la Fondation nationale des sciences politiques, 2001.

- Melé P., Patrimoine et action publique au centre des villes mexicaines, Paris, Presse de la Sorbonne nouvelle-IHEAL, 1998.

- Monnet J., «El prisma del ambulantaje. Conceptualización del ambulantaje, de los vendedores a los clientes : un acercamiento a la métrópoli postfordista", Séminaire El Ambulantaje en la Ciudad de México, México, CEMCA-UNAM, 2006.
- Montaud J.-M., Deux approches macroéconomiques du secteur informel en Équateur, Bordeaux, CED-DT n ${ }^{\circ}$ 47, 2000.

- OcAmpo J.-A. et J. MARTin (dir.), Une décennie d'ombres et de lumières. L'Amérique latine et les Caraïbes dans les années 1990, Santiago du Chili, CEPALC-IHEAL, 2003.

- Organisation mondiale des dolanes (OMD), Point sur la lutte contre la contrefaçon. Rapport mondial, 2004, Bruxelles, OMD, 2006.

- Oficina Internacional del Trabajo (OIT), El trabajo decente y la economía informal, Ginebra, OIT, Informe IV, 2002.

- Rivelois J., «Drogue, corruption et métamorphoses politiques (application à une comparaison Mexique-Brésil)", Revue Tiers Monde, t. XL, $\mathrm{n}^{\circ} 158,1999$.

- Rivelois J., "La défense des espaces urbains centraux par les acteurs de la marge», Séminaire Réinvestir le centre. Politiques de requalification, transformations urbaines et pratiques citadines dans les grandes villes d'Amérique latine, Paris, CREDAL-CRNS-IRD-Université Paris-III, 2006.

- Roman Reyes R. P., "Participación familiar en micronegocios en México», Tesis de doctorado, El Colegio de México, 2006.

- Roubaud F., L'économie informelle au Mexique. De la sphère domestique à la dynamique macroéconomique, Paris, Karthala-Orstom, 1994.

- Salas C. et E. ZePeda, «Ocupación e ingresos en México : 2000-2004 ", in E. de la Garza et C. SAlas (coord.), La situación del trabajo en México, México, Plaza y Valdés, 2006.

- Simón N., «Prácticas desleales e ilegales de comercio internacional en las industrias de textiles y prendas de vestir en México", in I. Rueda Peiros, N. Simón et M. L. GonzÁlez (coord.), La industría de la confección en México y China ante la globalización, México, UNAM-Porrúa, 2004.

- Soto H. de, E. Ghersi, M. Ghibellini et Ild, El otro sendero : la revolución informal, México, Editorial Diana, 1987.

- Wilson F., De la casa al taller, Zamora, El Colegio de Michoacán, 1990. 